\title{
Retrorectal cystic hamartoma: this cyst has a "tail" to tell
}

A 50-year-old man presented with a 6-week history of lumbosacral back pain and altered bowel habit without hematochezia. Abdominopelvic computed tomography $(\mathrm{CT})$ revealed a $5-\mathrm{cm}$ rectal mass with obliteration of the fat plane between the mass and the tip of the coccyx that was interpreted as representing a neoplasm ( $\bullet$ Fig. 1 a). Following a normal digital rectal exam, colonoscopy revealed a subtle posterior mid-rectal wall indentation, suspected to represent either an intramural process or extrinsic compression. The overlying mucosa appeared normal ( Fig. 1 b). A curvilinear endoscopic ultrasound examination excluded a subepithelial lesion, instead revealing a $3.4 \times$ $3.3 \mathrm{~cm}$ multiloculated cystic mass extrin- sic but adjacent to the rectal wall with no evidence of lymphadenopathy. This lesion was located distal and posterior to the inferior border of the prostate gland and comprised approximately 15 loculi. Each locule was thin-walled in the absence of septations. Many of the loculi contained intracystic echogenic material ( Video 1). Surgical resection of the retrorectal cystic mass mandated posterior dissection of the coccyx laterally. Surgical pathology confirmed a multiloculated presacral cystic mass measuring $6.0 \times$ $4.3 \times 3.2 \mathrm{~cm}$ ( Fig. $1 \mathrm{c}$ ). The cystic loculi were lined by squamous epithelium, transitional type epithelium, and columnar epithelium with focal goblet cells and no evidence of malignancy ( Fig. $1 \mathrm{~d}$ ). The findings were diagnostic of cystic hamartoma (tailgut cyst).

Until adverse event data for lower gastrointestinal endoscopic ultrasound (EUS) become available, the current recommendation is to avoid fine-needle aspiration (FNA) of perirectal cysts due to concerns regarding abscess formation even with prophylactic antibiotic use, as highlighted in one patient who subsequently required percutaneous drainage [1]. Complete intact surgical excision is advised to avoid the potential risk of needle-tract seeding, infection, and fistula formation [2].

Endoscopy_UCTN_Code_CCL_1AF_2AH

\section{Competing interests: None}

\section{Video 1}

A multiloculated extramural cystic structure with occasional intracystic locular echogenic material suggestive of mucin or debris.
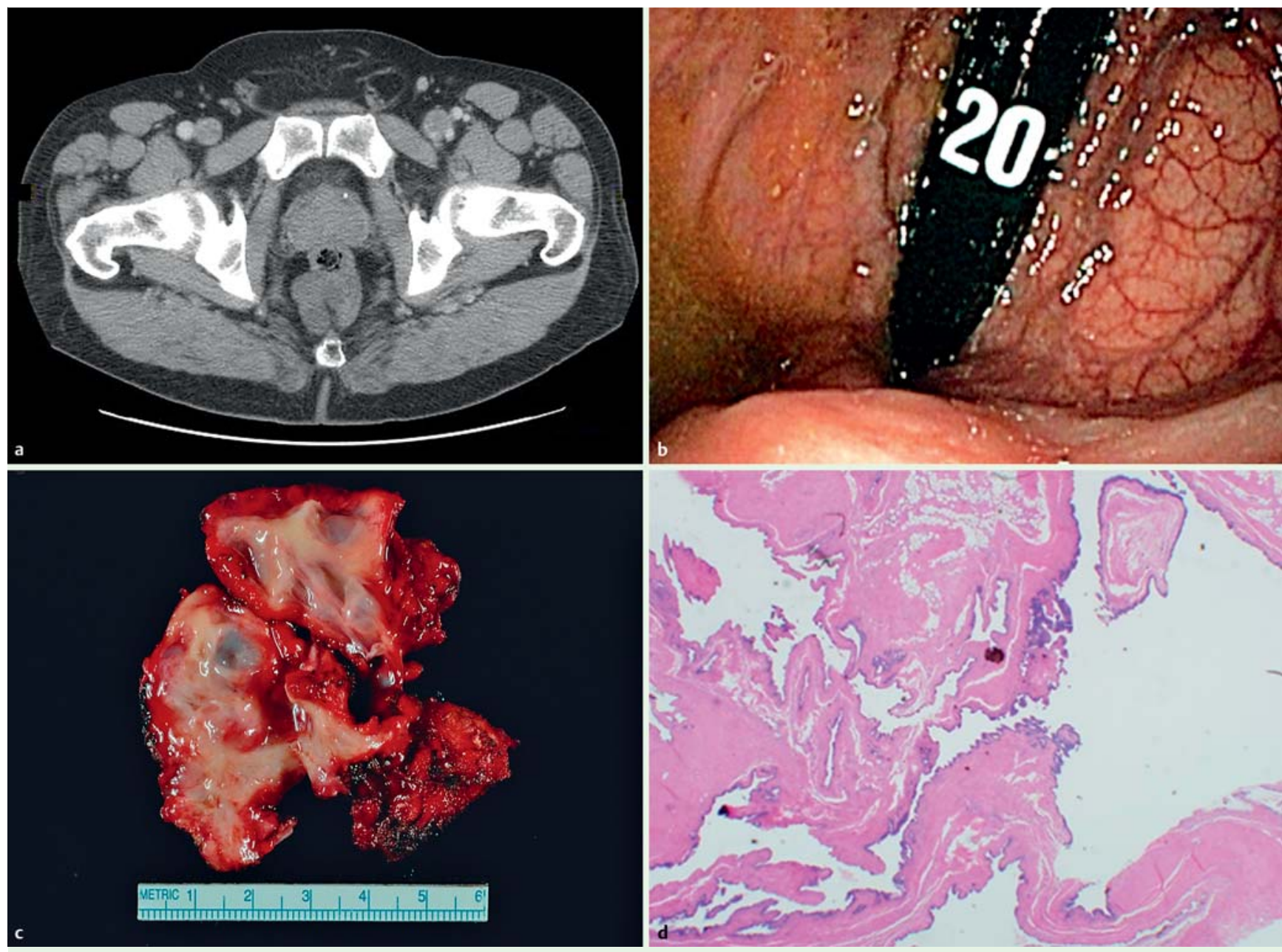

Fig. 1 a Preoperative computed tomography (CT) scan of the pelvis in a 50-year-old man with lumbosacral back pain and altered bowel habit without hematochezia. b Retroflexed endoscopic view of the presented lesion preoperatively. c, $\mathbf{d}$ The surgical and histopathological examination findings. 


\section{F. C. Gleeson, M. J. Levy}

Division of Gastroenterology and Hepatology, Mayo Clinic College of Medicine, Rochester, Minnesota, United States of America

\section{References}

1 Mohamadnejad M, Al-Haddad MA, Sherman $S$ et al. Utility of EUS-guided biopsy of extramural pelvic masses. Gastrointest Endosc 2012; 75: 146-151

2 Mathis KL, Dozois EJ, Grewal MS et al. Malignant risk and surgical outcomes of presacral tailgut cysts. Br J Surg 2010; 97: 575-579
Bibliography

Dol http://dx.doi.org/

10.1055/s-0033-1344130

Endoscopy 2013; 45: E191-E192

(c) Georg Thieme Verlag KG

Stuttgart · New York

ISSN 0013-726X
Corresponding author

\section{F. C. Gleeson}

Division of Gastroenterology and Hepatology Mayo Clinic College of Medicine 200 First Street SW

Rochester

MN 55905

USA

Fax: +507-266-3939

gleeson.ferga@mayo.edu 